OPEN ACCESS

Edited by:

Francisco Ciruela,

University of Barcelona, Spain

Reviewed by:

Peter Halasz,

Hungarian Society for Sleep

Medicine, Hungary

Vadim Valerievich Grubov,

Saratov State Technical University,

Russia

*Correspondence:

Miroslaw Latka

miroslaw.latka@pwr.edu.pl

Specialty section

This article was submitted to

Neuropharmacology,

a section of the journal

Frontiers in Neuroscience

Received: 25 July 2019

Accepted: 24 February 2020

Published: 13 March 2020

Citation:

Glaba P, Latka M, Krause MJ, Kuryło M, Jernajczyk W, Walas W and West BJ (2020) Changes in Interictal Pretreatment and Posttreatment EEG

in Childhood Absence Epilepsy.

Front. Neurosci. 14:196.

doi: 10.3389/fnins.2020.00196

\section{Changes in Interictal Pretreatment and Posttreatment EEG in Childhood Absence Epilepsy}

\author{
Pawel Glaba', Miroslaw Latka ${ }^{1 *}$, Małgorzata J. Krause ${ }^{2}$, Marta Kuryło', \\ Wojciech Jernajczyk ${ }^{3}$, Wojciech Walas ${ }^{4}$ and Bruce J. West ${ }^{5}$
}

${ }^{1}$ Department of Biomedical Engineering, Wrocław University of Science and Technology, Wrocław, Poland, ${ }^{2}$ Department of Pediatric Neurology, T. Marciniak Hospital, Wrocław, Poland, ${ }^{3}$ Clinical Neurophysiology, Institute of Psychiatry and Neurology, Warszawa, Poland, ${ }^{4}$ Neonatal and Pediatric Intensive Care Unit, University Hospital in Opole, Opole, Poland, ${ }^{5}$ Mathematics and Information Science Directorate, Army Research Office, Durham, NC, United States

Spike and wave discharges (SWDs) are a characteristic manifestation of childhood absence epilepsy (CAE). It has long been believed that they unpredictably emerge from otherwise almost normal interictal EEG. Herein, we demonstrate that pretreatment closed-eyes theta and beta EEG wavelet powers of CAE patients (20 girls and 10 boys, mean age $7.4 \pm 1.9$ years) are much higher than those of age-matched healthy controls at multiple sites of the 10-20 system. For example, at the C4 site, we observed a 100 and 63\% increase in power of theta and beta rhythms, respectively. We were able to compare the baseline and posttreatment wavelet power in 16 patients. Pharmacotherapy brought about a statistically significant decrease in delta and theta wavelet power in all the channels, e.g., for C4 the reduction was equal to $45 \%$ (delta) and $63 \%$ (theta). The less pronounced attenuation of posttreatment beta waves was observed in 13 channels (36\% at C4 site). The beta and theta wavelet power were positively correlated with the percentage of time in seizure (defined as the ratio of the duration of all absences which patients experienced to the duration of recording) for majority of channels. We hypothesize that the increased theta and beta powers result from cortical hyperexcitability and propensity for epileptic spike generation, respectively. We argue that the distinct features of CAE wavelet power spectrum may be used to define an EEG biomarker which could be used for diagnosis and monitoring of patients.

Keywords: childhood absence epilepsy, EEG, wavelets, biomarker, cortical excitability

\section{INTRODUCTION}

Childhood absence epilepsy (CAE) is the most common pediatric epileptic syndrome. It has a prevalence of $10-15 \%$ in childhood epilepsies and an incidence of 1.3-6 per 100,000 in children under the age of 16 years (Covanis, 2010). The disorder is most likely multifactorial, resulting from interactions between genetic and acquired factors (Gallentine and Mikati, 2012).

The ictal EEG of a typical absence seizure demonstrates rhythmic $\sim 3 \mathrm{~Hz}$ bilateral, synchronous and symmetrical spike and wave discharges (SWDs) with a median duration of approximately $10 \mathrm{~s}$, which may appear several times per day, sometimes as often as dozens of times per day (Schomer and Lopes da Silva, 2018). It has long been believed that SWDs are unpredictable and emerge from 
otherwise almost normal interictal EEG (Lopes da Silva et al., 2003). Interictal EEG abnormalities include sparse fragments of SWDs and focal discharges as well as posterior bilateral delta activity (Sadleir et al., 2006).

The response to monotherapy (valproic acid, ethosuximide, and lamotrigine) is generally good. As many as $75-85 \%$ of treated patients are seizure free and have a normal EEG (Covanis, 2010). The rest usually respond to a combination of drugs such as valproic acid and ethosuximide. Pharmacological seizure control with acceptable side effects is achieved for slightly more than half of the children. The relationship between pretreatment EEG and response to therapy is unknown (Dlugos et al., 2013). In general, CAE carries a good prognosis. Seizures spontaneously cease with ongoing maturation.

The cortical focus theory postulates that an absence seizure originates from a focus in the somatosensory cortex which is in a specific state conducive to seizure propagation. At the beginning of the seizure, it is the cortex that drives the thalamus, but prominent generalized spike-wave discharges result from their subsequent mutual interaction (Meeren et al., 2005). Considering the principal role of the cortex in absence seizure generation, we hypothesize that in CAE, interictal EEG manifests distinct features that can be detected even in routine, rest EEG recordings.

\section{MATERIALS AND METHODS}

We retrospectively analyzed a routine, anonymized pretreatment video EEG recording (average duration $37 \pm 13 \mathrm{~min}$ ) of $n=30$ CAE patients (20 girls and 10 boys) with a mean age of $7.4 \pm 1.9$ years. The analysis was approved by the ethics committee and the board of the T. Marciniak Hospital in Wrocław, Poland. CAE epilepsy syndrome was established based on of history, age at onset, clinical, and EEG findings as well as neuroimaging. In this cohort, we observed 173 seizures, $6 \pm 3$ per patient on average. The median seizure duration was $12 \pm 4$ s. On average, the ratio of the duration of all absences which patients experienced to the duration of EEG recording was $4 \pm 2 \%$. We refer to this ratio as the percentage of time in seizure (PTS). In nine cases, the first seizure occurred during hyperventilation, on average $130 \pm 55 \mathrm{~s}$ after its onset. The control group (15 girls and 15 boys) was age matched $(7.3 \pm 1.9$ years $)$ to the patients. EEG was recorded using the Elmiko Digitrack system with a BRAINTRONICS B.V. ISO-1032CE amplifier. The sampling frequency was equal to $250 \mathrm{~Hz}$. The 10-20 international standard was used to position $19 \mathrm{Ag} / \mathrm{AgCl}$ electrodes (impedances were below $5 \mathrm{k} \Omega$ ). The ground electrode was placed at the patients' forehead. The average reference electrode montage was used for time-frequency calculations. All the EEG recordings were performed between 2008 and 2018 by the same certified EEG technician.

After diagnosis, the pharmacotherapy of 16 out of 30 patients was administered by the outpatient neurology department of T. Marciniak Hospital. Valproic acid (two daily dosages of $244 \pm 58 \mathrm{mg}$ and $311 \pm 111 \mathrm{mg}$ ) was used in $75 \%$ of the cases and $19 \%$ of the patients were treated with ethosuximide (two daily dosages of $250 \mathrm{mg}$ ). A combination of valproic acid (250 mg twice a day) and ethosuximide (150 mg twice a day) was used in 6\% of the patients. The treatment response was evaluated with routine EEG with hyperventilation and photostimulation.

For each patient we extracted five closed-eyes (CE) motion artifact free pretreatment EEG segments: interictal (preceding the seizure by $226 \pm 122 \mathrm{~s}$ ), preictal A (beginning $31 \mathrm{~s}$ before the seizure), preictal B (beginning $16 \mathrm{~s}$ before the seizure), postictal A (beginning $1 \mathrm{~s}$ after seizure), and postictal B (beginning $16 \mathrm{~s}$ after the seizure). The average length of the interictal segments was: $84 \pm 32 \mathrm{~s}$. Due to the presence of motion artifacts and/or eye openings the length of the preictal and postictal segments slightly varied. Their average length was equal to $15 \pm 2 \mathrm{~s}, 15 \pm 3 \mathrm{~s}$, $16 \pm 1 \mathrm{~s}$, and $14 \pm 2 \mathrm{~s}$ for preictal A, preictal B, postictal A, and postictal B, respectively. The chosen length of pre- and postictal segments assured that data from all subjects could be included in analysis. For the controls, the average length of selected CE EEG segments was $55 \pm 27 \mathrm{~s}$. We also analyzed the most recent followup EEG which on average was recorded $1.5 \pm 2$ years after the video EEG used for diagnosis. The average length of CE segments selected for analysis from the follow-up EEG was $28 \pm 15 \mathrm{~s}$. There were no seizures in the follow-up EEGs.

We calculated the continuous wavelet transform (CWT) of EEG using the complex Morlet mother function. We analyzed the statistical properties of instantaneous wavelet power $w\left(f, t_{0}\right)$ (square of the modulus of complex wavelet coefficients) and temporal average $W(f)=\left\langle w\left(f, t_{0}\right)\right\rangle t_{0}$ over the selected EEG segment. We used the mother function with the center frequency $f_{c}=1$ and bandwidth $f_{b}=1.8$ (Latka et al., 2003). For these parameters, the transform was computed for three frequencies $f$ : 6.5, 9, and $15 \mathrm{~Hz}$. Figure 1A elucidates the rationale for such a choice of frequencies. The dashed red line in this figure represents the percentage difference between the $C 4$ wavelet spectrum $W(f)$ of patients and controls. The difference curve has two distinct local maxima at 6.5 and $15 \mathrm{~Hz}$. These frequencies were used to analyze theta and beta rhythms, respectively. The spectra of patients and controls have the maxima around $9 \mathrm{~Hz}$ and consequently such frequency was used for analysis of alpha waves. Figure 1B shows that the chosen wavelet calculated for $15 \mathrm{~Hz}$ may be used for detection of epileptic spikes. In other words, we focus on this part of the beta band which may be involved in the genesis of epileptic spikes. The spectacular increase of beta wavelet power in the vicinity of the epileptic spikes in Figure 1B can be employed in the simple but highly effective SWD detector which we describe elsewhere. The wavelet with $f_{c}=1$ and bandwidth $f_{b}=1.5$ calculated for frequency $3 \mathrm{~Hz}$ was used to quantify delta waves.

We used the Shapiro-Wilk test to determine whether the analyzed data were normally distributed. We compared the pretreatment and posttreatment values of $W(f)$ as well as the values of the controls with the Kruskal-Wallis test (with Tukey's post-hoc comparisons). The same test was used to analyze the changes in wavelet power in preictal and postictal segments. The patients' pretreatment wavelet power averaged over all channels was compared with that of controls using also a nonparametrical statistical testing (Maris and Oostenveld, 2007). The reported $p$-values for this test correspond to $1 \times 10^{5}$ random 

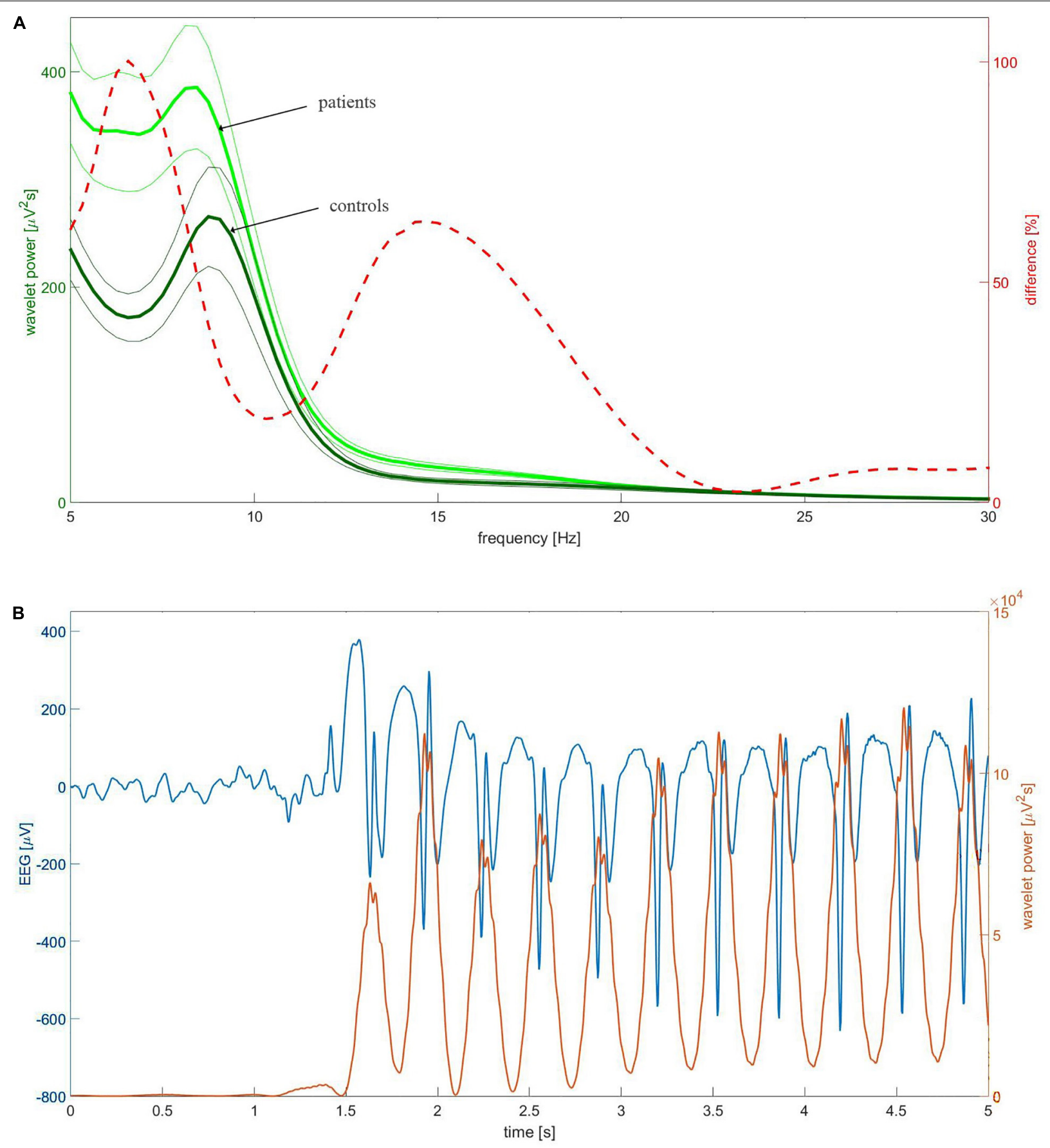

FIGURE 1 | (A) The group-averaged wavelet power spectrum in C4 channel of patients (thick light green) and controls (thick dark green). The complex Morlet wavelet $\left(f_{C}=1, f_{b}=1.8\right)$ was used as a mother function. The thin lines which bracket the averages represent standard error of the mean (SEM). The percentage difference between the patients and controls was drawn with the red dashed line. (B) Example of childhood absence epilepsy seizure. The presented section of EEG (blue curve) was extracted from EEG of a 7-year-old girl (patient BZ). The instantaneous power of the complex Morlet wavelet $\left(f_{C}=1, f_{b}=1.8\right)$ calculated for $15 \mathrm{~Hz}$ was drawn with the red curve.

draws. The area under the receiver operating characteristic curve (AUC) was used to quantify differences in $W(f)$ between patients and controls. AUC was also calculated for pretreatment and posttreatment values of $W$. The values of AUC, sensitivity and specificity were reported for selected channels. The correlation between the values of $W(f)$ for all four EEG bands and the percentage of time in seizure was examined for all 19 EEG channels using Pearson's correlation coefficient. The significance threshold for all the statistical tests was set to 0.05 . The wavelet and statistical analyses were done with MATLAB R2015A. 
We employed classifiers implemented in Weka software (version 3.8$)^{1}$ to discriminate between patients and controls using theta and beta wavelet power $W(f)$. Out of 38 such attributes, we used only these with non-zero gain ratio (entropic measure). Machine learning was performed with 10 -fold cross-validation.

\section{RESULTS}

Figure 2 shows that the pretreatment interictal closed-eyes theta and beta EEG wavelet powers of CAE patients are much higher than those of age-matched controls at multiple sites of the 1020 system. For the theta band, we observed a $100 \%$ increase in patients' wavelet power $W$ at C4 site $\left(343 \pm 296 \mu \mathrm{V}^{2} \mathrm{~s}\right.$ vs. $172 \pm 118 \mu \mathrm{V}^{2} \mathrm{~s}, p=9 \times 10^{-3}, \mathrm{AUC}=0.87$, sensitivity $=0.87$, specificity $=0.63$ ). For the beta band, the power in the patients was $63 \%$ greater than in the controls $\left(32 \pm 18 \mu \mathrm{V}^{2} \mathrm{~s}\right.$ vs. $20 \pm 13 \mu \mathrm{V}^{2} \mathrm{~s}, p=4 \times 10^{-3}, \mathrm{AUC}=0.77$, sensitivity $=0.77$, specificity $=0.73$ ). With the exception of channel $\mathrm{Fz}$, there was no statistically significant difference between the pretreatment patients and the controls for alpha rhythm. For the delta band, the increased pretreatment wavelet power was observed only at the occipital channels.

The value of $W(f)$ averaged over all 19 EEG channels was higher for pretreatment patients both for theta and beta bands. The corresponding $p$-values for Kruskall-Wallis test were equal to $4 \times 10^{-3}$ and $1 \times 10^{-3}$. For non-parametric testing, $p=8 \times 10^{-4}$ and $p=1 \times 10^{-4}$ for theta and beta rhythms, respectively. Kruskall-Wallis test did not detect differences in alpha and delta power between untreated patients and controls. Nonparametric testing showed the differences in the delta band with $p=2 \times 10^{-2}$.

In Figure 3 we present the probability density function (PDF) of instantaneous interictal theta and beta wavelet power $w\left(f, t_{0}\right)$ in channel C4 for the controls (light green) and the pretreatment patients (dark green). For both cohorts, we aggregated the values of $w\left(f, t_{0}\right)$ from all their members. It is apparent that the tail of the PDF is much longer for the pretreatment cohort. The insets in both subplots show the boxplots of time-averaged values of the pretreatment and posttreatment wavelet power $W$ as well as that of the controls. It is apparent from Figure $\mathbf{3 A}$ that pharmacotherapy suppressed the elevated pretreatment theta power that dropped $63 \%$ from $343 \pm 296 \mu \mathrm{V}^{2}$ s to $127 \pm 123$ $\mu \mathrm{V}^{2}$ s with post-hoc $p=2 \times 10^{-4}$ (AUC $=0.87$, sensitivity $=0.75$, specificity $=0.87)$. The patients' posttreatment $\mathrm{C} 4$ theta power was not statistically different from that of the controls $(p=0.26)$. Figure 3B shows that the influence of treatment on $\mathrm{C} 4$ beta power was weaker. In that case the power was reduced $36 \%$ from $32 \pm 18$ $\mu \mathrm{V}^{2}$ s to $21 \pm 22 \mu \mathrm{V}^{2}$ s with post-hoc $p=3 \times 10^{-3}$ (AUC $=0.78$, sensitivity $=0.81$, specificity $=0.70$ ).

For the alpha band, there were no statistically significant differences between the wavelet power in interictal segments and those in preictal and postictal ones. The $W$ of theta and beta rhythms was statistically greater than the interictal value only in postical A segments (channels Fp1, Fp2, F7, F8, and

${ }^{1}$ https://www.cs.waikato.ac.nz/ml/weka/
Fp1, F7, respectively). Such increases in theta wavelet power were not observed in postictal B segments. For preictal B and postictal A segments (adjacent to the absence seizure) the delta wavelet power was statistically greater than the interictal values in all 19 EEG channels. For example, at the C4 site, patients' wavelet power in preictal B segment was $367 \%$ greater than in the controls $\left(1,259 \pm 1,973 \mu V^{2} s\right.$ vs. $269 \pm 136 \mu V^{2} s$, $p=3 \times 10^{-5}, \mathrm{AUC}=0.82$, sensitivity $=0.76$, specificity $=0.83$ ) and $212 \%$ greater than the average value in the interictal segments $403 \pm 276 \mu \mathrm{V}^{2} \mathrm{~s}\left(p=2 \times 10^{-2}, \mathrm{AUC}=0.82\right.$, sensitivity $=0.69$, specificity $=0.73)$. Postictal A wavelet power $W$ at $\mathrm{C} 4$ for the delta band was $651 \%$ greater than in the controls $(2,020 \pm 3,837$ $\mu \mathrm{V}^{2}$ s vs. $269 \pm 136 \mu \mathrm{V}^{2} \mathrm{~s}, p=1 \times 10^{-5}, \mathrm{AUC}=0.83$, sensitivity $=0.76$, specificity $=0.83)$ and $402 \%$ greater than that for interictal segments $\left(p=1 \times 10^{-2}\right.$, AUC $=0.74$, sensitivity $=0.69$, specificity $=0.77$ ). There were no statistically significant differences in the interictal and postictal B delta wavelet power. Thus, the increase in postictal theta and delta wavelet power subsides within $30 \mathrm{~s}$ after the absence seizure.

Both for the theta and beta bands, the interictal wavelet power $W$ was positively correlated with the percentage of time in seizure PTS in majority of channels. For theta waves, there were 17 such channels and the average value of Pearson's correlation coefficient $r$ was equal to $0.51 \pm 0.11$ (range $0.26-0.66$, maximum at T3 and minimum at P4). For beta rhythm, we found 16 channels where correlations were significant (average value $r=0.51$, range 0.19-0.66, maximum at $\mathrm{P} 3$ and minimum at $\mathrm{O} 2$ ). No statistically significant relationship between $W$ and PTS was observed for alpha waves.

One can see in Figure 4 that pharmacotherapy primarily attenuates the low-frequency part of EEG spectrum. For all the channels, the values of interictal posttreatment delta and theta power are smaller than the pretreatment ones. In particular, for the delta band, $W$ for C4 channel fell $45 \%$ from $394 \pm 280 \mu \mathrm{V}^{2}$ s to $218 \pm 328 \mu \mathrm{V}^{2} \mathrm{~s}\left(p=7 \times 10^{-3}\right.$, AUC $=0.90$, sensitivity $=0.75$, specificity $=0.84)$. The less pronounced decrease in posttreatment beta power was observed in 13 channels. The fact that there is no increase of posttreatment alpha wavelet power implies that the attenuation of theta rhythm does not result from EEG maturation but is caused by treatment.

For channel C4, the reduction of $W$ was observed in 100 and $81 \%$ patients for the theta and beta band, respectively. The data presented in Figures $4 \mathrm{E}, \mathrm{F}$ are representative of the downward trend in the wavelet power during pharmacotherapy. For theta rhythm, a reduction was observed in all patients for channel C4, and all but one in channels: F7, T5, T6, P3, P4, and Pz. Upon averaging of all channels, both for theta and beta waves the reduction of $W$ was observed in $94 \%$ cases.

Out of 38 potential attributes, only 13 had non-zero gain ratio. For theta rhythm: C4 (0.28), Cz (0.28), P3 (0.24), O1 (0.22), Fz (0.21), $\mathrm{Pz}(0.21), \mathrm{C} 3$ (0.20), For beta waves: $\mathrm{Pz}(0.29)$, P3(0.28), P4 (0.28), O1 (0.26), O2 (0.21), C4 (0.19). The Bayesian network classifier (with default parameters) turned out to be the most balanced. It achieved accuracy of 78\%, specificity $79 \%$, and sensitivity $77 \%$. The classification was based on only six channels: C4, Cz, Fz for theta band and Pz, P3, O1 for beta band. 


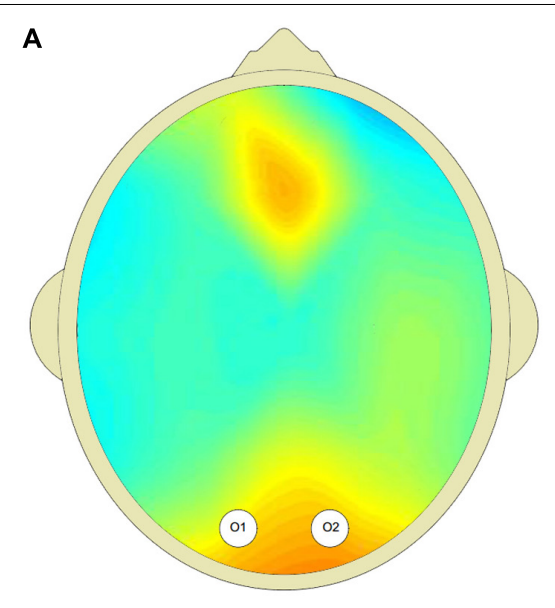

C

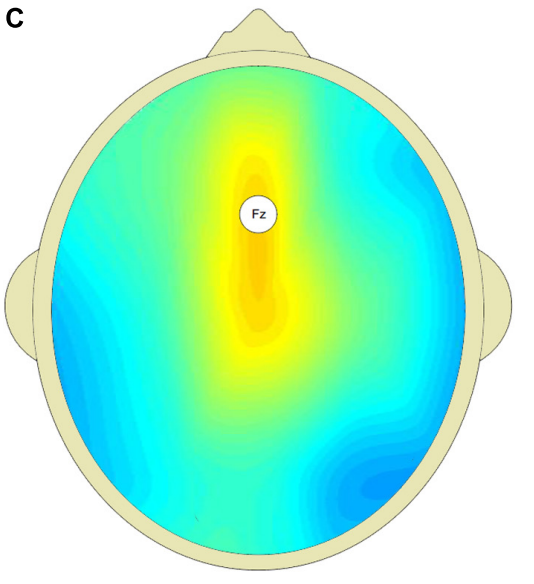

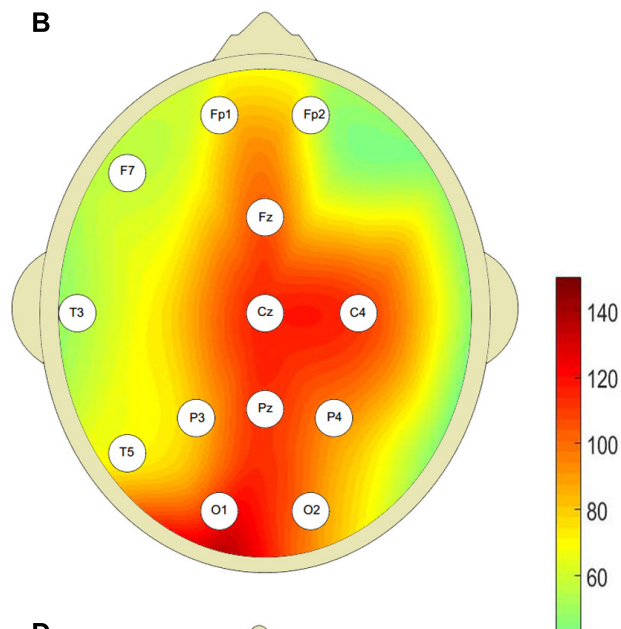

D

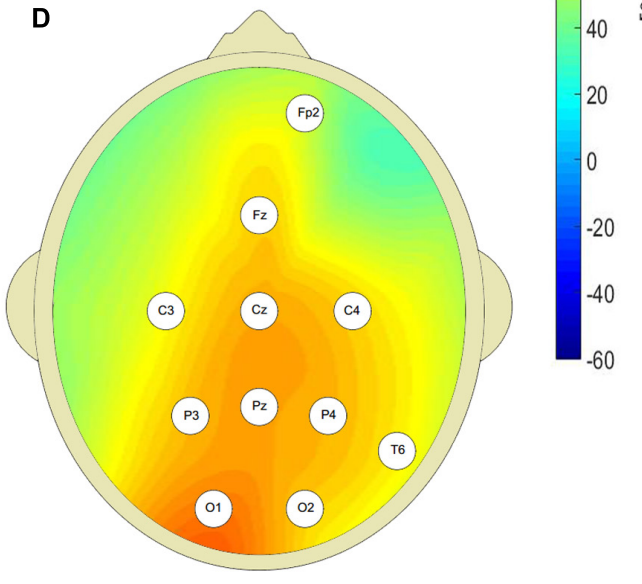

140
120
100
80
60

FIGURE 2 | Percentage differences between wavelet power of closed-eyes interictal pretreatment EEG of CAE patients and the controls (with respect to controls) for: delta (A), theta (B), alpha (C), and beta (D) rhythms. The EEG channel labels indicate sites for which the differences were statistically significant.

\section{DISCUSSION}

GAERS and the WAG/Rij rodent models have paved the way for the understanding of pathophysiology of human CAE seizures (Akman et al., 2010). Lüttjohann and Van Luijtelaar reviewed animal studies and the translation of research results from rodent models to humans (Lüttjohann and Van Luijtelaar, 2015). According to the cortical focus theory of Meeren et al. (2005) the genesis of absence seizure starts with a single spike which rapidly spreads over the hyperexcited cortex. The formation of prominent SWDs is possible only due to interaction with the thalamus which acts as a resonant circuit. The rapid generalization of spike-wave activity over the cortex is due to short-range intracortical fibers and to a subpopulation of cells that have long-range association fibers. These fibers run under the cortex in the white matter, making extensive connections with other cortical areas.

In rats, SWDs emerge from the cortical focus which is located either within the perioral somatosensory cortex (WAG/Rij) or the secondary somatosensory cortex (GAERS) and subsequently propagate via the corticothalamocortical loop. Polack et al. demonstrated that blockade of action potential discharge and synaptic activities in facial somatosensory cortical neurons (by topical application of tetrodotoxin) prevents the formation of SWDs (Polack et al., 2009). In contrast, pharmacological inhibition of a remote motor cortical region does not suppress ictal activities. Westmijse et al. discovered, using a beamforming source localization technique, that in humans the sources of spikes from a train of SWDs were at the frontal lateral, central and medial parietal cortices (Westmijse et al., 2009). The involvement of the thalamus in the generation of SWDs was demonstrated in combined EEG-fMRI (Granert et al., 2008; Moeller et al., 2010) and MEG (Tenney et al., 2013) studies. The differences between rat and human data are in the frequencies of SWDs $(6-11 \mathrm{~Hz}$ vs. $2.5-4 \mathrm{~Hz}$ ) and the location of the early local cortical activity which is quite variable in humans. The location may even change during a seizure (Westmijse et al., 2009) and is predominantly, but not exclusively, located in the frontal-central/parietal areas. The low variability of the position of cortical focus in the rats can easily be explained by the fact that both epileptic strains are fully inbred, and the animals are homozygous.

Herein, we found that the pretreatment closed-eyes theta wavelet power of CAE patients was much higher than that of age-matched controls at multiple sites of the 10-20 system. 

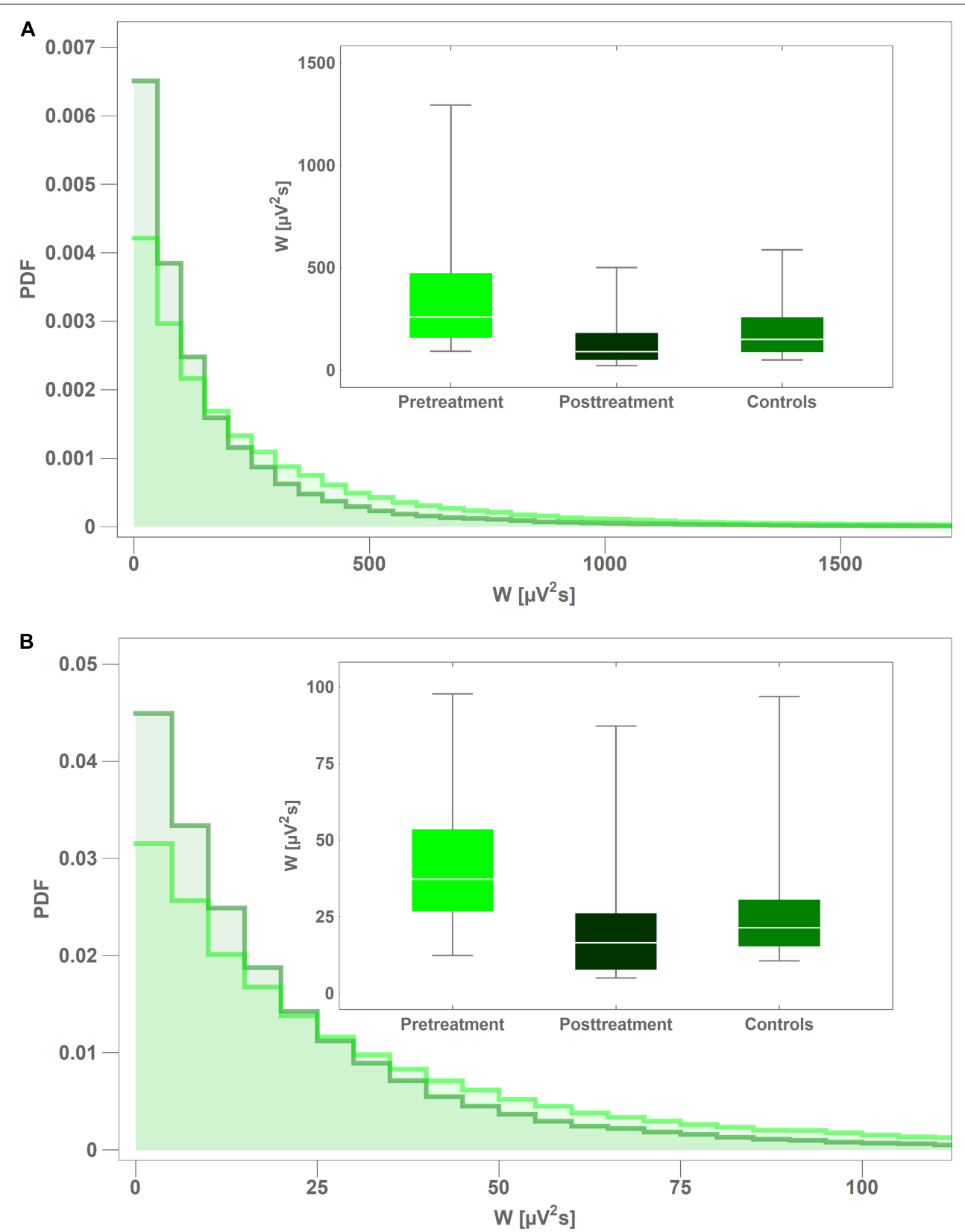

FIGURE 3 | Comparison of probability density function (PDF) of instantaneous wavelet power of interictal pretreatment EEG of CAE patients (light green) and the controls (dark green) for: theta (A) and beta (B) rhythms. PDFs were calculated from the closed-eyes segments extracted from C4 channel. The insets in both subplots show the boxplots of time-averaged values of the pretreatment and posttreatment wavelet power of as well as that of the controls.

Moreover, the theta wavelet power was positively correlated with the percentage of time in seizure. This result should not come as a surprise. In the late 1960s Doose et al. argued that strong rhythmic theta activity was an age-dependent electroencephalographic expression of a genetic disposition to convulsions, see a review paper (Doose and Baier, 1988) for a complete list of references. This hypothesis was noted, along with the opposing view, by the authors of the classic text on encephalography (Schomer and Lopes da Silva, 2018). To the best of our knowledge, initial observations have not been followed up on using quantitative EEG analysis (Doose et al., 2003). The more recent research provided new evidence for the role of theta rhythm in epilepsy. Clemens et al. (2012) using a Low Resolution Electromagnetic Tomography (LORETA) source localization found increased theta activity in the basal prefrontal and medial temporal limbic areas of CAE patients. Dömötör et al. (2017) observed reduced current source density in $0.5-8 \mathrm{~Hz}$ frequency range in CAE patients who responded to pharmacotherapy. Douw et al. (2010) in their MEG studies found that theta band brain connectivity and network topology is altered in epilepsy 


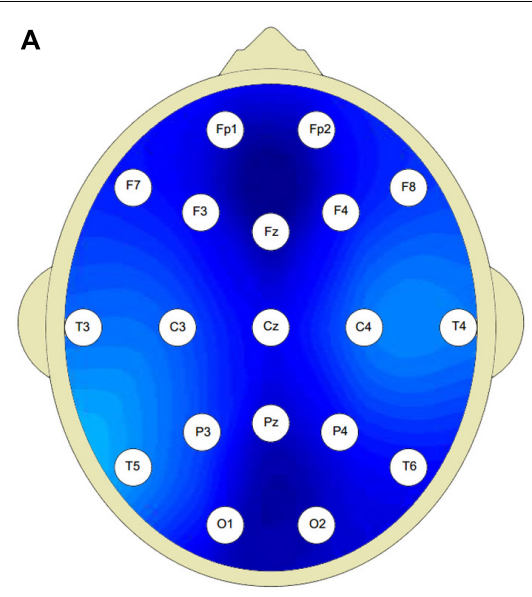

。
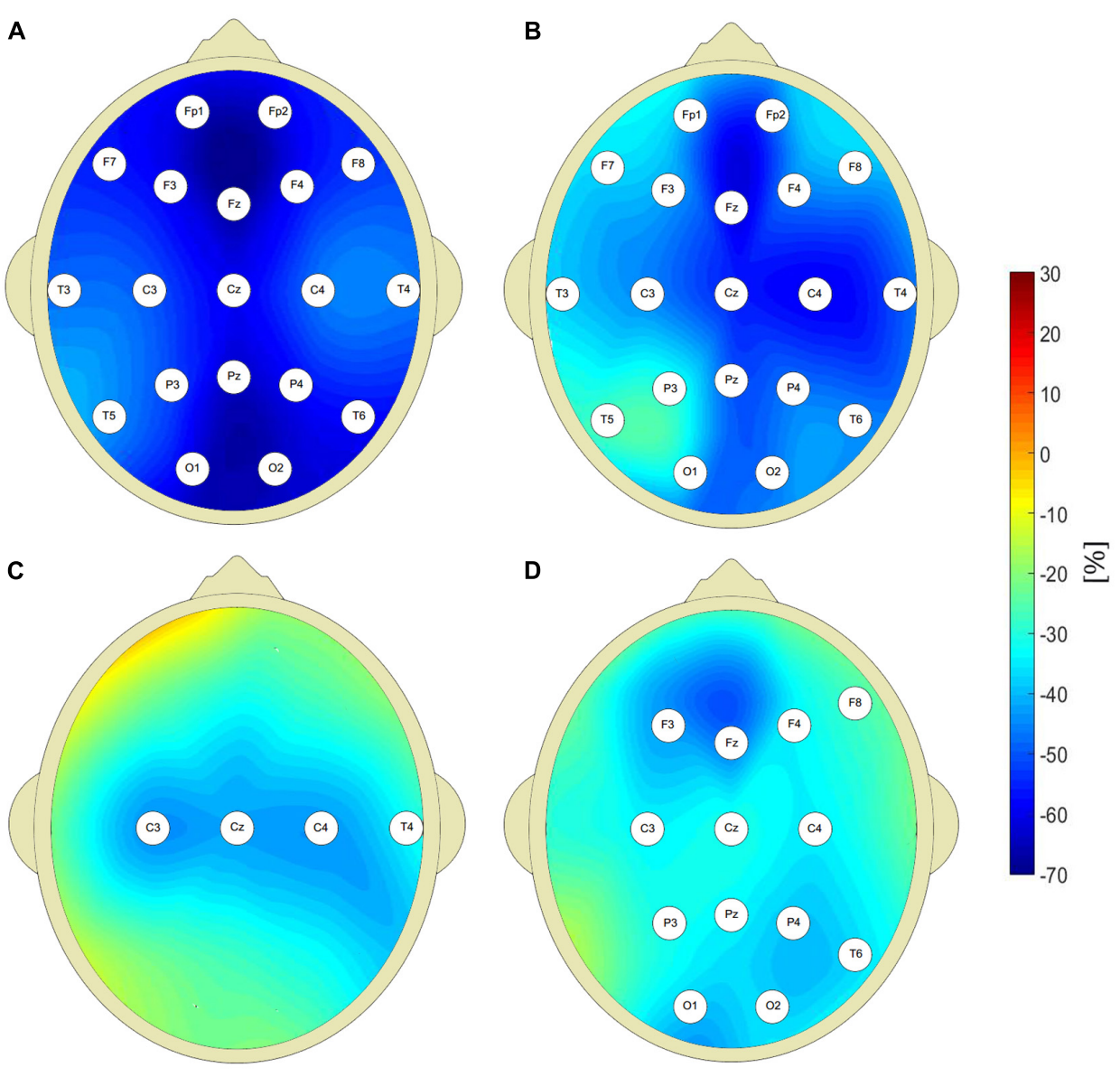

-Valproic acid

$\rightarrow$ Ethosuximide

-Combined

E

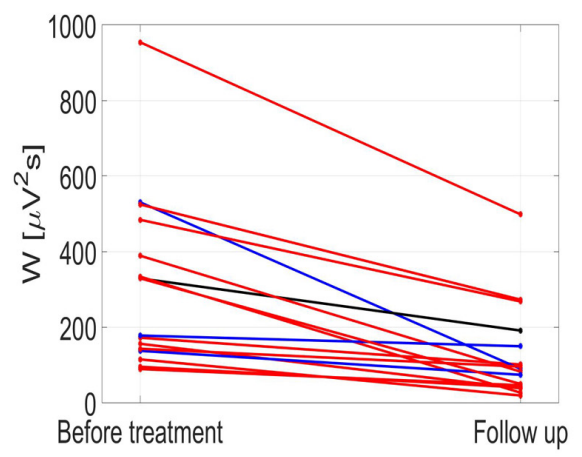

F

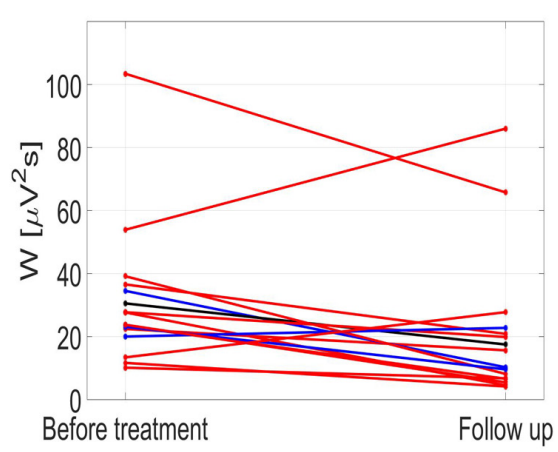

FIGURE 4 | Percentage change in posttreatment closed-eyes wavelet power of CAE patients with respect to the baseline for: delta (A), theta (B), alpha (C), and beta (D) rhythms. The EEG channel labels indicate sites for which the differences were statistically significant. Effect of treatment on $(\mathbf{E})$ theta and $(\mathbf{F})$ beta wavelet power $W$ in $C 4$ channel.

which developed in brain tumor patients. Milikovsky et al. (2017) discussed the properties of theta dynamics in the animal model of postinjury epilepsy. Sitnikova and van Luijtelaar (2009) found that the increased delta and theta power preceded SWDs in WAG/Rij rats.
We interpret the results presented herein as evidence in support of the following postulates. We hypothesize that the increased theta rhythm power in CAE patients is a manifestation of cortical hyperexcitability (Vestal and Blumenfeld, 2010). Since strong theta rhythm may be found in up to $15 \%$ of healthy 
children (Doose and Baier, 1988), cortical hyperexcitability may be a necessary but not a sufficient prerequisite for CAE. The increased beta power may reflect propensity for epileptic spikes generation - spikes that trigger absence seizures. This interpretation is corroborated by the recent work of Sorokin et al. (2016) who found that in rats absence seizure susceptibility correlates with pre-ictal beta oscillation. It is worth pointing out that as we detune the parameters of the analyzing wavelet away from the values optimal for spike detection, the differences in beta power between the pretreatment patients and controls disappear.

As with all epileptic syndromes, once the diagnosis of CAE is confirmed, only the absence or recurrence of seizures provides an indication as to whether anti-epileptic drugs (AEDs) have had any effect. We argue that the distinct features of CAE wavelet power spectrum may be used to define an EEG biomarker which could be used for diagnosis and long-term monitoring of patients. The most apparent applications of such monitoring would be in the assessment of the efficacy of pharmacotherapy and its duration.

Over the last three decades, transcranial magnetic stimulation (TMS) has become a principal tool for accessing cortical excitability associated with epilepsy (Bauer et al., 2014). However, there are essentially no TMS studies of drug-naive patients with CAE (Kessler, 2016). The barriers to the inclusion of children from such studies stem not only from the very nature of this experimental technique, e.g., its duration or immobilization of head during measurement, but also from a few fundamental reasons. For example, it is not clear whether the motor cortex physiology is a reliable marker of seizure susceptibility in children with less mature brain networks. Moreover, abnormalities in TMS markers of cortical excitability are not specific to epilepsy and may observed, among others, in ADHD, a common comorbid condition in children with epilepsy (Reilly et al., 2017). Future research should verify whether measurement of theta band power in CAE patients could be used in lieu of TMS. In particular, the previous TMS studies (Wright et al., 2006; Badawy et al., 2009) revealed that increased excitability preceded epileptic seizures. Thus, the question arises as to whether temporal changes in cortical excitability occur in CAE.

To use theta and beta wavelet power as biomarkers of CAE it needs to be proved that the observed changes between patient and controls are reproducible (which is important considering inherent variability of human EEG) and are not affected by the developmental changes of EEG which occur about the age of puberty. The main limitation of the study comes from its retrospective character. We analyzed the routine video EEGs that were used for diagnosis. We were able to demonstrate that the differences between patients and controls persisted throughout the EEG recordings. In particular, there were no differences in theta and beta wavelet power between the interictal segment (preceding the first observed seizure) and the preictal A, and postictal B segments. The data showed that the changes in wavelet power associated with absence seizure subsided within $30 \mathrm{~s}$ after its end. As we already mentioned, cortical excitability is known to vary with time. There is no doubt that future long-term EEG monitoring must verify our findings. The mean age of subjects was $7.4 \pm 1.9$ years. The follow-up EEGs were recorded on average one and a half year later. Puberty usually occurs in girls between the ages of 10 and 14, while in boys it generally occurs later. Thus, it is unlikely that the observed reduction of delta and theta power was merely effect of maturation and not brought about by pharmacotherapy. Moreover, there were no increases in alpha wavelet power in pretreatment and posttreatment EEGs which argues against the maturation effect.

Even though the analyzed pretreatment EEG segments were short, we were able to construct a balanced classifier which differentiated patients and controls with good accuracy using only several attributes. The assessment of applicability of such classifier in absence seizure diagnostics requires further studies with much larger datasets.

Pharmacological seizure control with acceptable side effects is achieved for slightly more than half of children with CAE. Herein we found that pharmacotherapy most effectively suppresses low-frequency EEG oscillations. Quantitative EEG analysis offers a unique opportunity to design a treatment which would more selectively attenuate the pathological theta and/or beta rhythms. The availability of low-cost, Internet connected personal EEG devices paves the way for home monitoring of patients which would facilitate drug titration and termination of pharmacotherapy.

\section{DATA AVAILABILITY STATEMENT}

The raw data supporting the conclusions of this article will be made available by the authors, without undue reservation, to any qualified researcher.

\section{ETHICS STATEMENT}

The studies involving human participants were reviewed and approved by the ethics committee of T. Marciniak Hospital in Wrocław, Poland. Written informed consent from the participants' legal guardian/next of kin was not required to participate in this study in accordance with the national legislation and the institutional requirements.

\section{AUTHOR CONTRIBUTIONS}

MJK, ML, and PG: conceptualization. PG, MK, MJK, and ML: investigation and original draft preparation. BW, WW, and $\mathrm{WJ}$ : review and editing. All authors contributed to methodology and formal analysis.

\section{ACKNOWLEDGMENTS}

MJK and ML dedicate this manuscript to the memory of our friend Andrzej Kozik who initiated this research project. This manuscript has been released as a Pre-Print at https://www. biorxiv.org/ (Glaba et al., 2019). 


\section{REFERENCES}

Akman, O., Demiralp, T., Ates, N., and Onat, F. Y. (2010). Electroencephalographic differences between WAG/Rij and GAERS rat models of absence epilepsy. Epilepsy Res. 89, 185-193. doi: 10.1016/j.eplepsyres.2009.12.005

Badawy, R., MacDonell, R., Jackson, G., and Berkovic, S. (2009). The peri-ictal state: cortical excitability changes within 24 h of a seizure. Brain 132, 1013-1021. doi: 10.1093/brain/awp017

Bauer, P. R., Kalitzin, S., Zijlmans, M., Sander, J. W., and Visser, G. H. (2014). Cortical excitability as a potential clinical marker of epilepsy: a review of the clinical application of transcranial magnetic stimulation. Int. J. Neural Syst. 24:1430001. doi: 10.1142/S0129065714300010

Clemens, B., Puskás, S., Besenyei, M., Emri, M., Opposits, G., Kis, S. A., et al. (2012). EEG-LORETA endophenotypes of the common idiopathic generalized epilepsy syndromes. Epilepsy Res. 99, 281-292. doi: 10.1016/j.eplepsyres.2011. 12.008

Covanis, A. (2010). “Childhood absence epilepsy," in Atlas of Epilepsies, ed. C. P. Panayiotopoulos (London: Springer), 1013-1023.

Dlugos, D., Shinnar, S., Cnaan, A., Hu, F., Moshé, S., Mizrahi, E., et al. (2013). Pretreatment EEG in childhood absence epilepsy: associations with attention and treatment outcome. Neurology 81, 150-156. doi: 10.1212/WNL. 0b013e31829a3373

Dömötör, J., Clemens, B., Puskás, S., and Fekete, I. (2017). Decrease of global current source density predicts successful treatment in absence and juvenile myoclonic epilepsies. Epilepsy Res. 133, 1-5. doi: 10.1016/j.eplepsyres.2017.03. 006

Doose, H., and Baier, W. K. (1988). Theta rhythms in the EEG: a genetic trait in childhood epilepsy. Brain Dev. 10, 347-354. doi: 10.1016/s0387-7604(88) 80091-3

Doose, H., Neubauer, B. A., and Neuhäuser, G. (2003). EEG in Childhood Epilepsy: Initial Presentation and Long-Term Follow-Up. France: John Libbey Eurotext.

Douw, L., van Dellen, E., de Groot, M., Heimans, J. J., Klein, M., Stam, C. J., et al. (2010). Epilepsy is related to theta band brain connectivity and network topology in brain tumor patients. BMC Neurosci. 11:103. doi: 10.1186/14712202-11-103

Gallentine, W. B., and Mikati, M. A. (2012). Genetic generalized epilepsies. J. Clin. Neurophysiol. 29, 408-419. doi: 10.1097/WNP.0b013e31826 bd92a

Glaba, P., Latka, M., Krause, M., Kuryło, M., Jernajczyk, W., Walas, W., et al. (2019). Changes in interictal pretreatment and posttreatment EEG in childhood absence epilepsy. bioRxiv [Preprint], doi: 10.1101/699868

Granert, O., Jansen, O., Stephani, U., Muhle, H., Wolff, S., Siniatchkin, M., et al. (2008). Simultaneous EEG-fMRI in drug-naive children with newly diagnosed absence epilepsy. Epilepsia 49, 1510-1519. doi: 10.1111/j.1528-1167. 2008.01626.x

Kessler, S. K. (2016). "Non-invasive brain stimulation in pediatric epilepsy: diagnostic and therapeutic uses," in Pediatric Brain Stimulation, eds A. Kirton, and D. L. Gilbert (Amsterdam: Elsevier), 281-304. doi: 10.1016/b978-0-12802001-2.00014-X

Latka, M., Was, Z., Kozik, A., and West, B. J. (2003). Wavelet analysis of epileptic spikes. Phys. Rev. Eng. 67:052902. doi: 10.1103/PhysRevE.67.05 2902

Lopes da Silva, F. H., Blanes, W., Kalitzin, S. N., Parra, J., Suffczynski, P., and Velis, D. N. (2003). Dynamical diseases of brain systems: different routes to epileptic seizures. IEEE Trans. Biomed. Eng. 50, 540-548. doi: 10.1109/TBME. 2003.810703

Lüttjohann, A., and Van Luijtelaar, G. (2015). Dynamics of networks during absence seizure's on- and offset in rodents and man. Front. Physiol. 6:16. doi: $10.3389 /$ fphys. 2015.00016
Maris, E., and Oostenveld, R. (2007). Nonparametric statistical testing of EEG- and MEG-data. J. Neurosci. Methods 164, 177-190. doi: 10.1016/j.jneumeth.2007.03. 024

Meeren, H., van Luijtelaar, G., Lopes da Silva, F., and Coenen, A. (2005). Evolving concepts on the pathophysiology of absence seizures: the cortical focus theory. Arch. Neurol. 62, 371-376. doi: 10.1001/archneur.62.3.371

Milikovsky, D. Z., Weissberg, I., Kamintsky, L., Lippmann, K., Schefenbauer, O., Frigerio, F., et al. (2017). Electrocorticographic dynamics as a novel biomarker in five models of epileptogenesis. J. Neurosci. 37, 4450-4461. doi: 10.1523/ jneurosci.2446-16.2017

Moeller, F., Gotman, J., Dubeau, F., Siniatchkin, M., Muhle, H., LeVan, P., et al. (2010). Absence seizures: individual patterns revealed by EEG-fMRI. Epilepsia 51, 2000-2010. doi: 10.1111/j.1528-1167.2010.02698.x

Polack, P.-O., Mahon, S., Chavez, M., and Charpier, S. (2009). Inactivation of the somatosensory cortex prevents paroxysmal oscillations in cortical and related thalamic neurons in a genetic model of absence epilepsy. Cereb. Cortex 19, 2078-2091. doi: 10.1093/cercor/bhn237

Reilly, C., Atkinson, P., Das, K. B., Chin, R. F. M., Aylett, S. E., Burch, V., et al. (2017). Parent- and teacher-reported symptoms of ADHD in school-aged children with active epilepsy: a population-based study. J. Atten. Disord. 21, 887-897. doi: 10.1177/1087054714558117

Sadleir, L. G., Farrell, K., Smith, S., Connolly, M. B., and Scheffer, I. E. (2006). Electroclinical features of absence seizures in childhood absence epilepsy. Neurology 67, 413-418. doi: 10.1212/01.wnl.0000228257.60184.82

Schomer, D. L., and Lopes da Silva, F. H. (2018). Niedermeyer's Electroencephalography: Basic Principles, Clinical Applications, and Related Fields, 7th Edn. Oxford: Oxford University Press.

Sitnikova, E., and van Luijtelaar, G. (2009). Electroencephalographic precursors of spike-wave discharges in a genetic rat model of absence epilepsy: power spectrum and coherence EEG analyses. Epilepsy Res. 84, 159-171. doi: 10.1016/ j.eplepsyres.2009.01.016

Sorokin, J., Paz, J. T., and Huguenard, J. R. (2016). Absence seizure susceptibility correlates with pre-ictal beta oscillations. J. Physiol. Paris 110, 372-381. doi: 10.1016/j.jphysparis.2017.05.004

Tenney, J. R., Fujiwara, H., Horn, P. S., Jacobson, S. E., Glauser, T. A., and Rose, D. F. (2013). Focal corticothalamic sources during generalized absence seizures: a MEG study. Epilepsy Res. 106, 113-122. doi: 10.1016/j.eplepsyres.2013.05.006

Vestal, M., and Blumenfeld, H. (2010). "Pathophysiology of absence seizures," in Atlas of Epilepsies, ed. C. P. Panayiotopoulos (London: Springer), 225-234. doi: 10.1007/978-1-84882-128-6-30

Westmijse, I., Ossenblok, P., Gunning, B., and van Luijtelaar, G. (2009). Onset and propagation of spike and slow wave discharges in human absence epilepsy: a MEG study. Epilepsia 50, 2538-2548. doi: 10.1111/j.1528-1167.2009.02162.x

Wright, M.-A. S. Y., Orth, M., Patsalos, P. N., Smith, S. J. M., and Richardson, M. P. (2006). Cortical excitability predicts seizures in acutely drug-reduced temporal lobe epilepsy patients. Neurology 67, 1646-1651. doi: 10.1212/01.wnl. 0000242729.85335.a3

Conflict of Interest: The authors declare that the research was conducted in the absence of any commercial or financial relationships that could be construed as a potential conflict of interest.

Copyright (c) 2020 Glaba, Latka, Krause, Kuryło, Jernajczyk, Walas and West. This is an open-access article distributed under the terms of the Creative Commons Attribution License (CC BY). The use, distribution or reproduction in other forums is permitted, provided the original author(s) and the copyright owner(s) are credited and that the original publication in this journal is cited, in accordance with accepted academic practice. No use, distribution or reproduction is permitted which does not comply with these terms. 